Supporting information for

\title{
Decarboxylative Claisen rearrangement reactions of allylic tosylmalonate esters
}

Donald Craig, Fabienne Grellepois

\section{General experimental:}

Dichloromethane $\left(\mathrm{CH}_{2} \mathrm{Cl}_{2}\right)$ was distilled from calcium hydride and all reactions were carried out with dry, freshly distilled solvents, under anhydrous conditions $\left(\mathrm{N}_{2}\right.$ atmosphere).

All reactions were monitored by thin-layer chromatography using precoated aluminiumbacked plates (Merck Kieselgel 60 F254) and are visualised by UV light and/or vaporisation of a solution of $\mathrm{KMnO}_{4}$. Merck Kieselgel silica gel (230-400 mesh) was used for flash chromatography.

Infra-red spectra were recorded on a Perkin 881 spectrophotometer. NMR spectra were recorded in $\mathrm{CDCl}_{3}$ on Jeol GX-270a spectrometer and calibrated using residual isotopic solvent as an internal reference. The following abbreviations are used to indicate the multiplicities: s, singlet; bs, broad singlet; d, doublet; t, triplet; q, quartet; quint, quintet; m, multiplet. Mass spectra were recorded using a micromass Platform II instrument operating in chemical ionisation (ammonia) mode. Elemental analysis were performed at the analytical laboratories of the University of North London.

- preparation of methyl malonate

- general procedure for the preparation of allylic malonate esters $\mathbf{3 a - j}$

- preparation of allylic methyl (toluene-4-sulfonyl)malonates $\mathbf{4 a - j}$

- decarboxylative Claisen rearrangement reactions (preparation of $\mathbf{5 a - c , 5 e - j}$ ) 
Preparation of methyl malonate: ${ }^{1}$ A mixture of Meldrum's acid and methanol (1 equiv.) was heated at $80^{\circ} \mathrm{C}$ for $20 \mathrm{~h}$. Evaporation of the residual acetone gave methyl malonate (quantitative yield) which was used directly in the next step without further purification.

General procedure for the preparation of allylic malonate esters 3a-j: To a solution of methyl malonate and the allylic alcohol (1 equiv.) in $\mathrm{CH}_{2} \mathrm{Cl}_{2}$, were added at $0{ }^{\circ} \mathrm{C}, 1,3$ dicyclohexylcarbodiimide (1 equiv.) and 4-(dimethylamino)pyridine (0.1 equiv.). After $18 \mathrm{~h}$ of stirring at $\mathrm{rt}$, the mixture was filtered through Celite and the residue washed with brine. The combined filtrate and washings were dried $\left(\mathrm{MgSO}_{4}\right)$ and concentrated under reduced pressure. Chromatography on silica gel $(25 \rightarrow 50 \%$ ethyl acetate-petroleum ether) of the residue gave allylic malonate esters $\mathbf{3 a - j}$.

\section{General procedure for the preparation of allylic methyl (toluene-4-sulfonyl)malonates} 4a-j: To a solution of allylic methyl malonates 3a-j (4.5 equiv.) in DMSO (c $\geq 2 \mathrm{~mol} \mathrm{~L}^{-1}$ ) was added very slowly, at $\mathrm{rt}$ and under $\mathrm{N}_{2}$, potassium tert-butoxide (4 equiv. of a $1.0 \mathrm{M}$ solution in $\mathrm{THF}^{2}$ ). The reaction was then stirred for $15 \mathrm{~min}$ before the addition of the tosyl fluoride (1 equiv.). After $20 \mathrm{~h}$ of stirring at $\mathrm{rt}$, the mixture was poured into aqueous $\mathrm{HCl}$ $(10 \%)$, extracted twice with ether and the combined organic layers were washed with brine, dried $\left(\mathrm{MgSO}_{4}\right)$ and concentrated under reduced pressure. Chromatography on silica gel $(25 \rightarrow 50 \%$ ethyl acetate-petroleum ether) of the residual mixture gave recovered excess allylic methyl malonates $\mathbf{3 a - j}$, followed by the desired allylic methyl (toluene-4sulfonyl)malonates $\mathbf{4 a - j}$. Yields cited for $\mathbf{4}$ are based on tosyl fluoride; yields cited for recovered $\mathbf{3}$ are calculated from the total recoverable amount based on the yield of $\mathbf{4}$ and the amount of 3 used (4.5 equiv.). For example, a $70 \%$ yield of 4 means the total recoverable amount of 3 is $(4.5-0.7)=3.8$ equiv; $74 \%$ recovery means that $(0.74 \times 3.8=2.81$ equiv. $)$ of 3 was recovered

\footnotetext{
${ }^{1}$ Brooks, D. W.; Castro de Lee, N.; Peevey, R. Tetrahedron Lett. 1984, 25, 4623-4626.

${ }^{2}$ Purchased from Aldrich Chemical Company.
} 


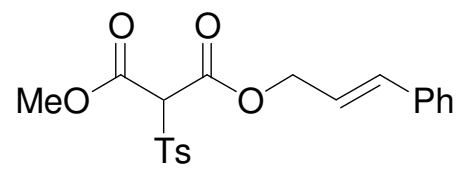

According to the general procedure, malonate 3a (5.98 g, $25.5 \mathrm{mmol}, 4.5$ equiv.) was tosylated with $t \mathrm{BuOK}(2.55 \mathrm{~g}, 22.7 \mathrm{mmol}, 4$ equiv.) and tosyl fluoride (988 mg, $5.67 \mathrm{mmol}, 1$ equiv.) in DMSO (6 mL). Chromatography $(25 \rightarrow 50 \%$ ethyl acetate-petroleum ether) yielded excess malonate 3a (3.72 g, 74\% recovery) and then (E)-methyl (3-phenyl-2-propenyl) (toluene-4-sulfonyl)malonate $4 \mathbf{a}(1.55 \mathrm{~g}, 70 \%)$ as a pale yellow oil; $\mathrm{R}_{\mathrm{f}} 0.37$ (25\% ethyl acetate-petroleum ether); IR (film) $v_{\mathrm{CO}} 1739 \mathrm{~cm}^{-1} ;{ }^{1} \mathrm{H} \mathrm{NMR} \delta 7.83(2 \mathrm{H}, \mathrm{d}, J 8.5 \mathrm{~Hz}, 2 \mathrm{H}-$ ortho Ts), 7.30 (7H, m, 2H-meta Ts, 5H Ph), $6.63(1 \mathrm{H}, \mathrm{d}, J 16.0 \mathrm{~Hz}, \mathrm{CH}=\mathrm{CH}-\mathrm{Ph}), 6.16(1 \mathrm{H}$, $\left.\mathrm{td}, J 6.5,16.0 \mathrm{~Hz}, \mathrm{OCH}_{2}-\mathrm{CH}=\mathrm{CH}\right), 5.00(1 \mathrm{H}, \mathrm{s}, \mathrm{CH}-\mathrm{Ts}), 4.80\left(2 \mathrm{H}, \mathrm{td}, J 6.5,16.0 \mathrm{~Hz}, \mathrm{OCH}_{2}\right)$, $3.79\left(3 \mathrm{H}, \mathrm{s}, \mathrm{OCH}_{3}\right), 2.37\left(3 \mathrm{H}, \mathrm{s}, \mathrm{CH}_{3} \mathrm{Ts}\right) ;{ }^{13} \mathrm{C} \mathrm{NMR} \delta 161.5,160.9,146.2,135.9,135.7$, 134.2, 130.2, 129.6, 128.8, 128.5, 126.8, 121.3, 74.5, 67.5, 53.8, 21.8; MS (CI) m/z: 406 $\left[\mathrm{M}+\mathrm{NH}_{4}\right]^{+}, 334,117,52$; HMRS (CI) $\mathrm{m} / \mathrm{z}$ calcd for $\left[\mathrm{C}_{20} \mathrm{H}_{20} \mathrm{O}_{6} \mathrm{~S}+\mathrm{NH}_{4}\right]^{+}:$406.1324, found: 406, 1324; Anal. calcd for $\mathrm{C}_{20} \mathrm{H}_{20} \mathrm{O}_{6} \mathrm{~S}$ : C, 61.84; H, 5.19; found: C, 61.77; H, 5.14.

\section{Methyl (2-propenyl) (toluene-4-sulfonyl)malonate (4b)}<smiles>C=CCOC(=O)C([18F])C(=O)OC</smiles>

According to the general procedure, malonate $3 \mathbf{b}$ (2.17 g, $13.7 \mathrm{mmol}, 4.5$ equiv.) was tosylated with $t \mathrm{BuOK}(1.37 \mathrm{~g}, 12.2 \mathrm{mmol}, 4$ equiv.) and tosyl fluoride (531 mg, $3.05 \mathrm{mmol}, 1$ equiv.) in DMSO (6 mL). Chromatography $(25 \rightarrow 50 \%$ ethyl acetate-petroleum ether) yielded excess 3b (1.45 g, 79\% recovery) and then methyl (2-propenyl) (toluene-4-sulfonyl)malonate 4b (640 mg, 67\%) as a colorless oil; $\mathrm{R}_{\mathrm{f}} 0.44$ (25\% ethyl acetate-petroleum ether); IR (film) $\mathrm{v}_{\mathrm{CO}} 1743 \mathrm{~cm}^{-1}$; ${ }^{1} \mathrm{H}$ NMR $\delta 7.83$ (2H, d, J 8.0 Hz, 2H-ortho Ts), 7.34 (2H, d, J 8.0 Hz, 2Hmeta Ts), $5.86\left(1 \mathrm{H}, \mathrm{m}, \mathrm{CH}=\mathrm{CH}_{2}\right), 5.30(1 \mathrm{H}, \mathrm{d}, J 18.0 \mathrm{~Hz}, \mathrm{CH}=\mathrm{CHaHb}), 5.25(1 \mathrm{H}, \mathrm{d}, J 10.5$ $\mathrm{Hz}, \mathrm{CH}=\mathrm{CHaHb}), 4.98(1 \mathrm{H}, \mathrm{s}, \mathrm{CH}-\mathrm{Ts}), 4.63\left(2 \mathrm{H}, \mathrm{d}, J 6.0 \mathrm{~Hz}, \mathrm{OCH}_{2}\right), 3.76\left(3 \mathrm{H}, \mathrm{s}, \mathrm{OCH}_{3}\right)$, 2.43 (3H, s, $\left.\mathrm{CH}_{3} \mathrm{Ts}\right) ;{ }^{13} \mathrm{C}$ NMR $\delta 161.4,160.6,146.0,134.0,130.3,130.0,129.4,119.4,74.2$, 67.2, 53.5, 21.5; MS (CI) m/z: $330\left[\mathrm{M}+\mathrm{NH}_{4}\right]^{+}, 176,139,108 ;$ HRMS (CI) m/z calcd for 
$\left[\mathrm{C}_{14} \mathrm{H}_{16} \mathrm{O}_{6} \mathrm{~S}+\mathrm{NH}_{4}\right]^{+}:$330.1011, found: 330.1010; Anal. calcd for $\mathrm{C}_{14} \mathrm{H}_{16} \mathrm{O}_{6} \mathrm{~S}: \mathrm{C}, 53.84 ; \mathrm{H}$, 5.04; found: C, 53.71; H, 5.04.

\section{(E)-Methyl (2-pentenyl) (toluene-4-sulfonyl)malonate (4c)}

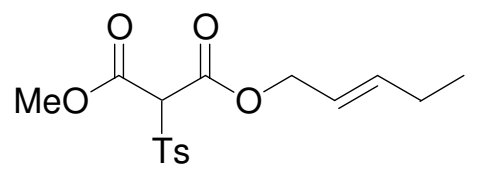

According to the general procedure, malonate 3c (2.03 g, $10.9 \mathrm{mmol}, 4.5$ equiv.) was tosylated with $t \mathrm{BuOK}(1.16 \mathrm{~g}, 10.4 \mathrm{mmol}, 4$ equiv.) and tosyl fluoride (453 mg, $2.60 \mathrm{mmol}, 1$ equiv.) in DMSO (5.5 mL). Chromatography $(25 \rightarrow 50 \%$ ethyl acetate-petroleum ether), yielded the excess malonate 3c (1.04 g, 65\% recovery) and then (E)-methyl (2-pentenyl) (toluene-4-sulfonyl)malonate $\mathbf{4 c}(531 \mathrm{mg}, 60 \%)$ as a colorless oil; $\mathrm{R}_{\mathrm{f}} 0.55$ (25\% ethyl acetatepetroleum ether); IR (film) $v_{\mathrm{CO}} 1745 \mathrm{~cm}^{-1} ;{ }^{1} \mathrm{H} \mathrm{NMR} \delta 7.84(2 \mathrm{H}, \mathrm{d}, J 8.0 \mathrm{~Hz}, 2 \mathrm{H}$-ortho Ts), $7.34(2 \mathrm{H}, \mathrm{d}, J 8.0 \mathrm{~Hz}, 2 \mathrm{H}-m e t a \mathrm{Ts}), 5.81\left(1 \mathrm{H}, \mathrm{td}, J 6.5,15.0 \mathrm{~Hz}, \mathrm{OCH}_{2}-\mathrm{CH}=\mathrm{CH}\right), 5.47(1 \mathrm{H}$, $\left.\operatorname{ttd}, J 1.5,6.5,15.0 \mathrm{~Hz}, \mathrm{CH}=\mathrm{CH}-\mathrm{CH}_{2}\right), 4.96(1 \mathrm{H}, \mathrm{s}, \mathrm{CH}-\mathrm{Ts}), 4.58\left(2 \mathrm{H}, \mathrm{d}, J 6.5 \mathrm{~Hz}, \mathrm{OCH}_{2}\right)$, $3.77\left(3 \mathrm{H}, \mathrm{s}, \mathrm{OCH}_{3}\right), 2.44\left(3 \mathrm{H}, \mathrm{s}, \mathrm{CH}_{3} \mathrm{Ts}\right), 2.05\left(2 \mathrm{H}\right.$, quint, $\left.J 7.5 \mathrm{~Hz}, \mathrm{CH}_{2}-\mathrm{CH}_{3}\right), 0.98(3 \mathrm{H}, \mathrm{t}, J$ $\left.7.5 \mathrm{~Hz}, \mathrm{CH}_{2}-\mathrm{CH}_{3}\right) ;{ }^{13} \mathrm{C} \mathrm{NMR} \delta 161.5,160.7,146.0,139.4,134.3,130.1,129.5,121.3,74.4$, 67.6, 53.6, 25.2, 21.7, 12.9; MS (CI) m/z : $358\left[\mathrm{M}+\mathrm{NH}_{4}\right]^{+}, 246,204,136,52 ; \mathrm{HRMS}(\mathrm{CI}) \mathrm{m} / \mathrm{z}$ calcd for $\left[\mathrm{C}_{16} \mathrm{H}_{20} \mathrm{O}_{6} \mathrm{~S}+\mathrm{NH}_{4}\right]^{+}: 358.1324$, found: 358.1320.); Anal. calcd for $\mathrm{C}_{16} \mathrm{H}_{20} \mathrm{O}_{6} \mathrm{~S}: \mathrm{C}$, 56.46; H, 5.92; found: C, 56.29; H, 6.01.

\section{(Z)-Methyl (2-pentenyl) (toluene-4-sulfonyl)malonate (4d)}

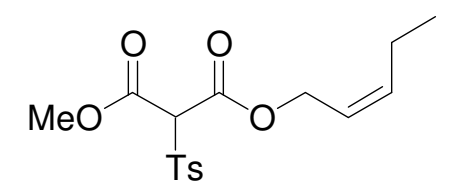

According to the general procedure, malonate 3d (1.34 g, $7.20 \mathrm{mmol}, 4.5$ equiv.) was tosylated with $t \mathrm{BuOK}(718 \mathrm{mg}, 6.40 \mathrm{mmol}, 4$ equiv.) and tosyl fluoride (279 mg, $1.60 \mathrm{mmol}$, 1 equiv.) in DMSO (3.5 mL). Chromatography $(25 \rightarrow 50 \%$ ethyl acetate-petroleum ether), yielded the excess malonate $\mathbf{3 d}(921 \mathrm{mg}, 89 \%$ recovery) and then (Z)-methyl (2-pentenyl) (toluene-4-sulfonyl)malonate $\mathbf{4 d}$ (409 mg, 75\%) as a colorless oil; $\mathrm{R}_{\mathrm{f}} 0.50$ (25\% ethyl acetatepetroleum ether); IR (film) $v_{\mathrm{CO}} 1741 \mathrm{~cm}^{-1} ;{ }^{1} \mathrm{H}$ NMR $\delta 7.81(2 \mathrm{H}, \mathrm{d}, J 8.0 \mathrm{~Hz}, 2 \mathrm{H}$-ortho Ts), 
$7.32(2 \mathrm{H}, \mathrm{d}, J 8.0 \mathrm{~Hz}, 2 \mathrm{H}-m e t a \mathrm{Ts}), 5.65(1 \mathrm{H}, \mathrm{m}, \mathrm{CH}=\mathrm{CH}), 5.38(1 \mathrm{H}, \mathrm{m}, \mathrm{CH}=\mathrm{CH}), 4.94(1 \mathrm{H}$, s, CH-Ts), $4.66\left(2 \mathrm{H}, \mathrm{d}, J 7.0 \mathrm{~Hz}, \mathrm{OCH}_{2}\right), 3.74\left(3 \mathrm{H}, \mathrm{s}, \mathrm{OCH}_{3}\right), 2.42\left(3 \mathrm{H}, \mathrm{s}, \mathrm{CH}_{3} \mathrm{Ts}\right), 2.05(2 \mathrm{H}$, quint, J 7.0 Hz, $\left.\mathrm{CH}_{2}-\mathrm{CH}_{3}\right), 0.93\left(3 \mathrm{H}, \mathrm{t}, J 7.0 \mathrm{~Hz}, \mathrm{CH}_{2}-\mathrm{CH}_{3}\right) ;{ }^{13} \mathrm{C} \mathrm{NMR} \delta$ 161.5, 160.9, 146.1, 138.6, 134.2, 130.2, 129.6, 121.1, 74.5, 62.7, 53.7, 21.8, 20.9, 14.0; MS (CI) m/z : 358 $\left[\mathrm{M}+\mathrm{NH}_{4}\right]^{+}$; HRMS (CI) $\mathrm{m} / \mathrm{z}$ calcd for $\left[\mathrm{C}_{16} \mathrm{H}_{20} \mathrm{O}_{6} \mathrm{~S}+\mathrm{NH}_{4}\right]^{+}: 358.1324$, found: 358.1331.); Anal. calcd for $\mathrm{C}_{16} \mathrm{H}_{20} \mathrm{O}_{6} \mathrm{~S}$ : C, 56.46; H, 5.92; found: C, 56.37; H, 5.95.

\section{Methyl (3-methyl-2-butenyl) (toluene-4-sulfonyl)malonate (4e)}

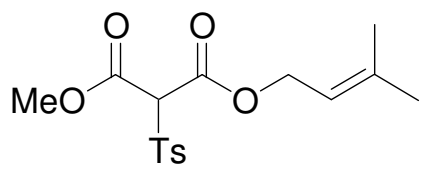

According to the general procedure, malonate $3 \mathrm{e}$ (1.94 g, $10.4 \mathrm{mmol}, 4.5$ equiv.) was tosylated with $t \mathrm{BuOK}$ (1.04 g, $9.24 \mathrm{mmol}, 4$ equiv.) and tosyl fluoride (403 mg, $2.31 \mathrm{mmol}, 1$ equiv.) in DMSO (5 mL). Chromatography ( $25 \rightarrow 50 \%$ ethyl acetate-petroleum ether), yielded the excess malonate $3 \mathbf{e}(1.37 \mathrm{~g}, 91 \%$ recovery) and then methyl (3-methyl-2-butenyl) (toluene4-sulfonyl)malonate $4 \mathbf{e}(707 \mathrm{mg}, 90 \%)$ as a colorless oil; $\mathrm{R}_{\mathrm{f}} 0.46$ (25\% ethyl acetatepetroleum ether); IR (film) $v_{\mathrm{CO}} 1744 \mathrm{~cm}^{-1} ;{ }^{1} \mathrm{H} \mathrm{NMR} \delta 7.83(2 \mathrm{H}, \mathrm{d}, J 8.5 \mathrm{~Hz}, 2 \mathrm{H}$-ortho Ts), $7.33(2 \mathrm{H}, \mathrm{d}, J 8.5 \mathrm{~Hz}, 2 \mathrm{H}-m e t a \mathrm{Ts}), 5.25\left(1 \mathrm{H}, \mathrm{m}, \mathrm{CH}=\mathrm{C}\left(\mathrm{CH}_{3}\right)_{2}\right), 4.94(1 \mathrm{H}, \mathrm{s}, \mathrm{CH}-\mathrm{Ts}), 4.63$ $\left(2 \mathrm{H}, \mathrm{d}, J 7.5 \mathrm{~Hz}, \mathrm{OCH}_{2}\right), 3.77\left(3 \mathrm{H}, \mathrm{s}, \mathrm{OCH}_{3}\right), 2.44\left(3 \mathrm{H}, \mathrm{s}, \mathrm{CH}_{3} \mathrm{Ts}\right), 1.74\left(3 \mathrm{H}, \mathrm{s}, \mathrm{CH}_{3}\right), 1.67$ $\left(3 \mathrm{H}, \mathrm{s}, \mathrm{CH}_{3}\right) ;{ }^{13} \mathrm{C}$ NMR $\delta 161.5,160.9,146.0,140.8,134.3,130.1,129.5,117.2,74.4,63.8$, 53.6, 25.8, 21.8, 18.0; MS (CI) m/z: $358\left[\mathrm{M}+\mathrm{NH}_{4}\right]^{+}$, 290, 246, 204, 174, 136, 86; HRMS (CI) $\mathrm{m} / \mathrm{z}$ calcd for $\left[\mathrm{C}_{16} \mathrm{H}_{20} \mathrm{O}_{6} \mathrm{~S}+\mathrm{NH}_{4}\right]^{+}: 358.1324$, found: 358.1331. Anal. calcd for $\mathrm{C}_{16} \mathrm{H}_{20} \mathrm{O}_{6} \mathrm{~S}: \mathrm{C}$, 56.46; H, 5.92; found: C, 56.36; H, 5.87.

\section{(E)-Methyl (2-methyl-3-phenyl-2-propenyl) (toluene-4-sulfonyl)malonate (4f)}<smiles>COC(=O)C([18F])C(=O)OC/C(C)=C/c1ccccc1</smiles>

According to the general procedure, malonate $3 \mathbf{f}$ (1.88 g, $7.57 \mathrm{mmol}, 4.5$ equiv.) was tosylated with $t \mathrm{BuOK}$ (754 mg, $6.72 \mathrm{mmol}, 4$ equiv.) and tosyl fluoride (293 mg, $1.68 \mathrm{mmol}, 1$ equiv.) in DMSO $(3.5 \mathrm{~mL})$. Chromatography $(25 \rightarrow 50 \%$ ethyl acetate-petroleum ether), yielded the excess malonate 3f (1.45 g, 99\% recovery) and then (E)-methyl (2-methyl-3-phenyl-2- 
propenyl) (toluene-4-sulfonyl)malonate $4 \mathbf{f}(380 \mathrm{mg}, 56 \%)$ as a colorless oil; $\mathrm{R}_{\mathrm{f}} 0.37$ (25\% ethyl acetate-petroleum ether); IR (film) $v_{\mathrm{CO}} 1744 \mathrm{~cm}^{-1} ;{ }^{1} \mathrm{H}$ NMR $\delta 7.85(2 \mathrm{H}, \mathrm{d}, J 8.0 \mathrm{~Hz}, 2 \mathrm{H}-$ ortho Ts), 7.27 (7H, m, 2H-meta Ts, 5H Ph), 6.49 (1H, s, C=CH-Ph), 5.05 (1H, s, CH-Ts), $4.71\left(2 \mathrm{H}, \mathrm{s}, \mathrm{OCH}_{2}\right), 3.77\left(3 \mathrm{H}, \mathrm{s}, \mathrm{OCH}_{3}\right), 2.37\left(3 \mathrm{H}, \mathrm{s}, \mathrm{CH}_{3} \mathrm{Ts}\right), 2.05\left(2 \mathrm{H}\right.$, quint, J 7.5 Hz, $\mathrm{CH}_{2-}$ $\left.\mathrm{CH}_{3}\right), 0.98\left(3 \mathrm{H}, \mathrm{t}, J 7.5 \mathrm{~Hz}, \mathrm{CH}_{2}-\mathrm{CH}_{3}\right), 1.82\left(3 \mathrm{H}, \mathrm{s}, \mathrm{CH}_{3}\right) ;{ }^{13} \mathrm{C} \mathrm{NMR} \delta 161.6,160.8,146.2$, $136.7,134.2,131.3,130.2,129.8,129.7,129.0,128.3,127.1,74.5,72.7,53.8,21.8,15.5 ; \mathrm{MS}$ (CI) $\mathrm{m} / \mathrm{z}: 420\left[\mathrm{M}+\mathrm{NH}_{4}\right]^{+}, 304,289,246,174,148,131 ;$ HRMS (CI) $\mathrm{m} / \mathrm{z}$ calcd for $\left[\mathrm{C}_{21} \mathrm{H}_{22} \mathrm{O}_{6} \mathrm{~S}+\mathrm{NH}_{4}\right]^{+}: 420.1481$, found: 420.1475; Anal. calcd for $\mathrm{C}_{21} \mathrm{H}_{22} \mathrm{O}_{6} \mathrm{~S}: \mathrm{C}, 62.67 ; \mathrm{H}$, 5.51; found: C, 62.55; H, 5.63 .

\section{(S)-(4-Isopropenyl-1-cyclohexenylmethyl) methyl (toluene-4-sulfonyl)malonate (4g)}

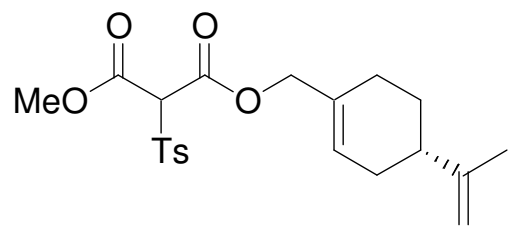

According to the general procedure, malonate $3 \mathrm{~g}$ (1.83 g, $7.25 \mathrm{mmol}, 4.5$ equiv.) was tosylated with $t \mathrm{BuOK}$ (724 mg, $6.45 \mathrm{mmol}, 4$ equiv.) and tosyl fluoride (280 mg, $1.61 \mathrm{mmol}$, 1 equiv.) in DMSO (3.5 mL). Chromatography $(25 \rightarrow 50 \%$ ethyl acetate-petroleum ether), yielded the excess malonate $3 \mathrm{~g}$ (1.53 g, 100\% recovery) and then (S)-(4-isopropenyl-1cyclohexenylmethyl) methyl (toluene-4-sulfonyl)malonate $\mathbf{4 g}(530 \mathrm{mg}, 81 \%)$ as a colorless oil; $\mathrm{R}_{\mathrm{f}} 0.48$ (25\% ethyl acetate-petroleum ether); IR (film) $v_{\mathrm{CO}} 1744 \mathrm{~cm}^{-1} ;{ }^{1} \mathrm{H}$ NMR $\delta 7.84(2 \mathrm{H}, \mathrm{d}$, $J 8.0 \mathrm{~Hz}, 2 \mathrm{H}$-ortho Ts), 7.34 (2H, d, J 8.0 Hz, 2H-meta Ts), 5.75 (1H, bs, CH=C), 4.97 (1H, s, CH-Ts), $4.70\left(2 \mathrm{H}, \mathrm{d}, J 4.5 \mathrm{~Hz}, \mathrm{CH}_{2}=\mathrm{C}\right), 4.53\left(2 \mathrm{H}, \mathrm{s}, \mathrm{OCH}_{2}\right), 3.77\left(3 \mathrm{H}, \mathrm{s}, \mathrm{OCH}_{3}\right), 2.44(3 \mathrm{H}, \mathrm{s}$, $\left.\mathrm{CH}_{3} \mathrm{Ts}\right), 2.20-1.72(6 \mathrm{H}, \mathrm{m}), 1.71\left(3 \mathrm{H}, \mathrm{s}, \mathrm{CH}_{3}\right), 1.41(1 \mathrm{H}, \mathrm{m}, \mathrm{CH}) ;{ }^{13} \mathrm{C}$ NMR $\delta$ 161.6, 160.9, $149.4,146.1,134.3,131.4,130.2,130.0,129.6,127.6,108.9,74.5,71.0,53.7,40.7,30.5$,

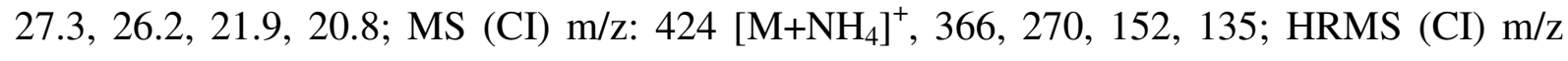
calcd for $\left[\mathrm{C}_{21} \mathrm{H}_{26} \mathrm{O}_{6} \mathrm{~S}+\mathrm{NH}_{4}\right]^{+}: 424.1794$, found: 424.1778; Anal. calcd for $\mathrm{C}_{21} \mathrm{H}_{26} \mathrm{O}_{6} \mathrm{~S}: \mathrm{C}$, 62.05; H, 6.45; found: C, 62.13; H, 6.39 . 
$(1 ' R$,

sulfonyl)malonate (4h)

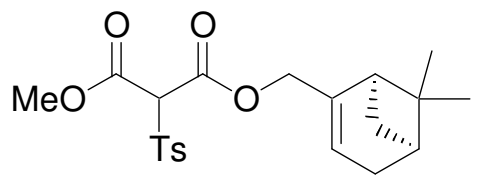

According to the general procedure, malonate $3 \mathbf{h}$ (1.95 g, $7.75 \mathrm{mmol}, 4.5$ equiv.) was tosylated with $t \mathrm{BuOK}(811 \mathrm{mg}, 7.22 \mathrm{mmol}, 4$ equiv.) and tosyl fluoride (300 mg, $1.72 \mathrm{mmol}$, 1 equiv.) in DMSO (3.8 mL). Chromatography $(25 \rightarrow 50 \%$ ethyl acetate-petroleum ether), yielded the excess malonate $\mathbf{3 h}(1.50 \mathrm{~g}, 99 \%$ recovery) and then ( 1 ' $\mathrm{R}, 5$ ' $\mathrm{R})-(6,6$-dimethylbicyclo[3.1.1]hept-2-en-2-yl) methyl (toluene-4-sulfonyl)malonate $4 \mathbf{h}(549 \mathrm{mg}, 78 \%$ ) as a colorless oil; $\mathrm{R}_{\mathrm{f}} 0.64$ (25\% ethyl acetate-petroleum ether); IR (film) $v_{\mathrm{CO}} 1745 \mathrm{~cm}^{-1} ;{ }^{1} \mathrm{H}$ NMR $\delta 7.84(2 \mathrm{H}, \mathrm{d}, J 8.0 \mathrm{~Hz}, 2 \mathrm{H}$-ortho Ts), $7.34(2 \mathrm{H}, \mathrm{d}, J 8.0 \mathrm{~Hz}, 2 \mathrm{H}-$ meta Ts$), 5.55$ (1H, s, $\left.\mathrm{C}=\mathrm{CH}-\mathrm{CH}_{2}\right), 4.95(1 \mathrm{H}, \mathrm{s}, \mathrm{CH}-\mathrm{Ts}), 4.50\left(2 \mathrm{H}, \mathrm{s}, \mathrm{OCH}_{2}\right), 3.76\left(3 \mathrm{H}, \mathrm{s}, \mathrm{OCH}_{3}\right), 2.44\left(3 \mathrm{H}, \mathrm{s}, \mathrm{CH}_{3}\right.$ Ts), $2.36(1 \mathrm{H}, \mathrm{m}, \mathrm{CH}), 2.24\left(2 \mathrm{H}, \mathrm{d}, J 8.0 \mathrm{~Hz}, \mathrm{CH}-\mathrm{CH}_{2}-\mathrm{CH}\right), 2.05\left(2 \mathrm{H}, \mathrm{m}, \mathrm{CH}_{2}\right), 1.25(3 \mathrm{H}, \mathrm{s}$, $\left.\mathrm{CH}_{3}\right), 1.10(1 \mathrm{H}, \mathrm{m}, \mathrm{CH}), 0.75\left(3 \mathrm{H}, \mathrm{d}, J 1.0 \mathrm{~Hz}, \mathrm{CH}_{3}\right) ;{ }^{13} \mathrm{C} \mathrm{NMR} \delta 161.5,160.9$ 146.0, 141.6, 134.4, 134.3, 130.1, 129.6, 123.2, 74.4, 69.5, 53.6, 43.4, 40.5, 38.0, 31.4, 31.3, 26.1, 21.8, 20.9; MS (CI) m/z: $424\left[\mathrm{M}+\mathrm{NH}_{4}\right]^{+}, 304,246,174,152,135$; HRMS (CI) m/z calcd for $\left[\mathrm{C}_{21} \mathrm{H}_{26} \mathrm{O}_{6} \mathrm{~S}+\mathrm{NH}_{4}\right]^{+}:$424.1794, found: 424.1785; Anal. calcd for $\mathrm{C}_{21} \mathrm{H}_{26} \mathrm{O}_{6} \mathrm{~S}: \mathrm{C}, 62.05 ; \mathrm{H}$, 6.45; found: C, 62.13; H, 6.45 .

( \pm )-(E)-Methyl (3-penten-2-yl) (toluene-4-sulfonyl)malonate (4i)

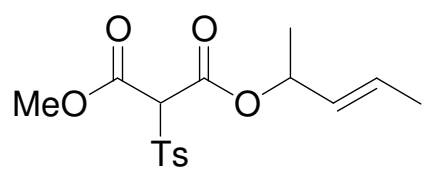

According to the general procedure, malonate $3 \mathbf{i}(2.33 \mathrm{~g}, 12.5 \mathrm{mmol}, 4.5$ equiv.) was tosylated with $t$ BuOK (1.25 g, $11.1 \mathrm{mmol}, 4$ equiv.) and tosyl fluoride (484 mg, $2.78 \mathrm{mmol}, 1$ equiv.) in DMSO (6 mL). Chromatography $(25 \rightarrow 50 \%$ ethyl acetate-petroleum ether), yielded the excess malonate $3 \mathbf{i}(1.50 \mathrm{~g}, 83 \%$ recovery) and then ( \pm )-(E)-methyl (3-penten-2-yl) (toluene-4sulfonyl)malonate $\mathbf{4 i}(750 \mathrm{mg}, 79 \%)$ as a colorless oil; $\mathrm{R}_{\mathrm{f}} 0.50$ (25\% ethyl acetate-petroleum ether); IR (film) $v_{\mathrm{CO}} 1742 \mathrm{~cm}^{-1}$; ${ }^{1} \mathrm{H}$ NMR $\delta 7.83(2 \mathrm{H}, \mathrm{d}, J 8.0 \mathrm{~Hz}, 2 \mathrm{H}$-ortho Ts), 7.33 (2H, d, 
$J 8.0 \mathrm{~Hz}, 2 \mathrm{H}-m e t a \mathrm{Ts}), 7.68(1 \mathrm{H}, \mathrm{m}, \mathrm{CH}=\mathrm{C}), 5.32(2 \mathrm{H}, \mathrm{m}, \mathrm{CH}=\mathrm{C}, \mathrm{OCH}), 4.92(1 \mathrm{H}, \mathrm{s}, \mathrm{CH}-$ Ts), $3.76\left(3 \mathrm{H}, \mathrm{s}, \mathrm{OCH}_{3}\right), 2.43\left(3 \mathrm{H}, \mathrm{s}, \mathrm{CH}_{3} \mathrm{Ts}\right), 1.66\left(3 \mathrm{H}, \mathrm{t}, J 6.0 \mathrm{~Hz}, \mathrm{CH}_{3}-\mathrm{CH}-\mathrm{O}\right), 1.26(3 \mathrm{H}$, $\left.\mathrm{dd}, J 2.5,6.0 \mathrm{~Hz}, \mathrm{CH}_{3}-\mathrm{CH}=\mathrm{CH}\right) ;{ }^{13} \mathrm{C}$ NMR $\delta 161.8,160.2,146.0,130.2,129.9,129.8,129.5$, 129.3, 74.8, 74.7, 53.6, 21.8, 20.0, 17.8; MS (CI) m/z: 698, $358\left[\mathrm{M}+\mathrm{NH}_{4}\right]^{+} ; \mathrm{HRMS}(\mathrm{CI}) \mathrm{m} / \mathrm{z}$ calcd for $\left[\mathrm{C}_{16} \mathrm{H}_{20} \mathrm{O}_{6} \mathrm{~S}+\mathrm{NH}_{4}\right]^{+}: 358.1324$, found: 358.1335; Anal. calcd for $\mathrm{C}_{16} \mathrm{H}_{20} \mathrm{O}_{6} \mathrm{~S}: \mathrm{C}$, 56.46; H, 5.92; found: C, 56.29; H, 5.79.

(1'R, 5'R)-(5-Isopropenyl-2-methyl-cyclohex-2-enyl) methyl (toluene-4-sulfonyl)malonate $(4 \mathbf{j})$

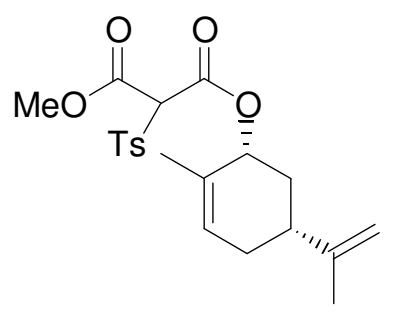

According to the general procedure, malonate $\mathbf{3 j}$ (3.16 g, $12.52 \mathrm{mmol}, 4.5$ equiv.) was tosylated with $t \mathrm{BuOK}(1.25 \mathrm{~g}, 11.13 \mathrm{mmol}, 4$ equiv.) and tosyl fluoride (485 mg, $2.78 \mathrm{mmol}$, 1 equiv.) in DMSO (6.2 mL). Chromatography $(25 \rightarrow 50 \%$ ethyl acetate-petroleum ether), yielded the excess malonate $3 \mathbf{j}$ (1.63 g, 66\% recovery) and then ( 1 'R, 5'R)-(5-isopropenyl-2methylcyclohex-2-enyl) methyl (toluene-4-sulfonyl)malonate $\mathbf{4 j}$ (838 $\mathrm{mg}, 74 \%$ ) as a colorless oil; $\mathrm{R}_{\mathrm{f}} 0.50$ (25\% ethyl acetate-petroleum ether); IR (film) $v_{\mathrm{CO}} 1743 \mathrm{~cm}^{-1} ;{ }^{1} \mathrm{H}$ NMR $\delta 7.86$ (2H, dd, J $2.08 .0 \mathrm{~Hz}, 2 \mathrm{H}$-ortho Ts), $7.34(2 \mathrm{H}, \mathrm{d}, J 8.0 \mathrm{~Hz}, 2 \mathrm{H}-m e t a \mathrm{Ts}), 5.59$ (1H, bs, $\mathrm{CH}=\mathrm{C}), 5.46(1 \mathrm{H}, \mathrm{bs}, \mathrm{CH}-\mathrm{OCO}), 4.97(1 \mathrm{H}, \mathrm{s}, \mathrm{CH}-\mathrm{Ts}), 4.69$ (2H, d, J $\left.12.0 \mathrm{~Hz}, \mathrm{CH}_{2}=\mathrm{C}\right), 3.78$ and $3.76\left(3 \mathrm{H}, 2 \mathrm{~s}, \mathrm{OCH}_{3}\right), 2.43\left(3 \mathrm{H}, \mathrm{s}, \mathrm{CH}_{3} \mathrm{Ts}\right), 2.30-1.80(4 \mathrm{H}, \mathrm{m}), 1.68\left(3 \mathrm{H}, \mathrm{s}, \mathrm{CH}_{3}\right), 1.56$ $\left(3 \mathrm{H}, \mathrm{d}, J 10.5 \mathrm{~Hz}, \mathrm{CH}_{3}\right), 1.43(1 \mathrm{H}, \mathrm{m}) ;{ }^{13} \mathrm{C} \mathrm{NMR} \delta 161.5,160.8,147.9,146.1,131.7,130.2$, 129.6, 127.0, 109.7, 76.9 and 76.8, 74.7 and 74.5, 53.7, 40.2, 33.4 and 33.3, 30.7, 21.8, 20.5, 18.7 and 18.6; MS (CI) m/z: 558, $424\left[\mathrm{M}+\mathrm{NH}_{4}\right]^{+}, 304,246,152,135 ; \mathrm{HRMS}(\mathrm{CI}) \mathrm{m} / \mathrm{z}$ calcd for $\left[\mathrm{C}_{21} \mathrm{H}_{26} \mathrm{O}_{6} \mathrm{~S}+\mathrm{NH}_{4}\right]^{+}$: 424.1794, found: 424.1786; Anal. calcd for $\mathrm{C}_{21} \mathrm{H}_{26} \mathrm{O}_{6} \mathrm{~S}: \mathrm{C}, 62.05 ; \mathrm{H}$, 6.45; found: C, 61.99; H, 6.47. 


\section{General procedure for decarboxylative Claisen rearrangement reactions:}

Using BSA-KOAc (Method A): To solutions of tosylmalonates $\mathbf{4 a - k}$ in $\mathrm{CH}_{2} \mathrm{Cl}_{2}$ were added, at $\mathrm{rt}$ and under $\mathrm{N}_{2}$, BSA (1 equiv.) and KOAC (0.1 equiv.). After stirring for the periods indicated, the reaction mixtures were concentrated under reduced pressure. Chromatography on silica gel afforded the decarboxylated rearranged products 5a-k.

Using TBDMSOTf-DBU (Method B): To solutions of tosylmalonates 4a-k in $\mathrm{CH}_{2} \mathrm{Cl}_{2}$ were added simultaneously, at $\mathrm{rt}$ and under $\mathrm{N}_{2}$, TBDMSOTf (2.1 equiv.) and DBU (2.1 equiv.). After 15-30 min of stirring, the reaction mixtures were concentrated under reduced pressure. Chromatography on silica gel afforded the decarboxylated rearranged products $\mathbf{5 a - k}$ Methyl 3-phenyl-(toluene-4-sulfonyl)pent-4-enoate (5a) ${ }^{3}$

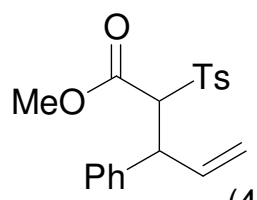

$(47: 53)$

Method A: According to the general procedure, a mixture of tosylmalonate $4 \mathbf{a}$ (168 mg, 0.43 mmol), BSA (105 $\mu \mathrm{L}, 0.43 \mathrm{mmol}, 1$ equiv.) and KOAc (4 mg, $0.04 \mathrm{mmol}, 0.1$ equiv.) in $\mathrm{CH}_{2} \mathrm{Cl}_{2}(5 \mathrm{~mL})$ was stirred for $4 \mathrm{~h}$ at $\mathrm{rt}$. Evaporation of solvent and chromatography of the residue (25\% ethyl acetate-petroleum ether) yielded methyl 3-phenyl-(toluene-4sulfonyl)pent-4-enoate $\mathbf{5 a}(120 \mathrm{mg}, 81 \%)$ as a colorless solid.

Method B: According to the general procedure, a mixture of tosylmalonate $4 \mathbf{a}$ (112 $\mathrm{mg}, 0.29$ mmol), TBDMSOTf (139 $\mu \mathrm{L}, 0.61 \mathrm{mmol}, 2.1$ equiv.) and DBU (90 $\mu \mathrm{L}, 0.61 \mathrm{mmol}, 2.1$ equiv.) in $\mathrm{CH}_{2} \mathrm{Cl}_{2}(1 \mathrm{~mL})$ was stirred for $15 \mathrm{~min}$. Evaporation of solvent and chromatography of the residue (25\% ethyl acetate-petroleum ether) yielded $\mathbf{5 a}(80 \mathrm{mg}, 81 \%)$ as a colorless solid.

$\mathrm{R}_{\mathrm{f}} 0.58$ (25\% ethyl acetate-petroleum ether); ${ }^{1} \mathrm{H}$ NMR $\delta 7.78(2 \mathrm{H}, \mathrm{d}, J 8.0 \mathrm{~Hz}, 2 \mathrm{H}$-ortho $\mathrm{Ts})$, $7.33\left(2 \mathrm{H}, \mathrm{d}, J 8.0 \mathrm{~Hz}, 2 \mathrm{H}-\mathrm{meta}_{\mathrm{Ts}}\right), 7.15(5 \mathrm{H}, \mathrm{m}, 5 \mathrm{H} \mathrm{Ph}), 6.12$ (ddd, J 2.0, 8.0, $10.5 \mathrm{~Hz}$, $\mathrm{CH}=\mathrm{CH}_{2}$ minor isomer), 5.85 (ddd, $J 1.5,9.0,10.0 \mathrm{~Hz}, \mathrm{CH}=\mathrm{CH}_{2}$ major isomer), $5.16(1 \mathrm{H}, \mathrm{d}$, $J 9.0 \mathrm{~Hz}, \mathrm{CH}=\mathrm{CHaHb}), 5.08(\mathrm{~d}, J 17.0 \mathrm{~Hz}, \mathrm{CH}=\mathrm{CHaHb}$ one isomer), $5.03(\mathrm{~d}, J 17.0 \mathrm{~Hz}$, $\mathrm{CH}=\mathrm{CHa} H b$ other isomer), 4.50 (d, $J 8.5 \mathrm{~Hz}, \mathrm{CH}$-Ts minor isomer), 4.45 (d, $J 8.5 \mathrm{~Hz}, \mathrm{CH}$-Ts major isomer), 4.16 (dd, $J$ 8.5, $9.5 \mathrm{~Hz}, \mathrm{CH}$-Ph major isomer), 4.05 (dd, $J$ 8.5, 9.5 Hz, CH-Ph

\footnotetext{
${ }^{3}$ Masuyama, Y.; Mitsunaga, Y.; Kurusu, Y.; Segawa, K. I. Bull. Chem. Soc. Japan 1987, 60, 3431-3432.
} 
minor isomer), 3.72 (s, $\mathrm{OCH}_{3}$ major isomer), $3.24\left(\mathrm{~s}, \mathrm{OCH}_{3}\right.$ minor isomer), $2.44\left(\mathrm{~s}, \mathrm{CH}_{3} \mathrm{Ts}\right.$ minor isomer), 2.34 (s, $\mathrm{CH}_{3}$ Ts major isomer).

\section{Methyl (toluene-4-sulfonyl)pent-4-enoate (5b) ${ }^{4}$}

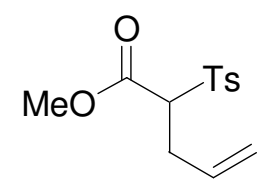

Method A: According to the general procedure, a mixture of tosylmalonate $\mathbf{4 b}$ (151 mg, 0.48 mmol) BSA (117 $\mu \mathrm{L}, 0.48 \mathrm{mmol}, 1$ equiv.) and KOAc (5 mg, $0.05 \mathrm{mmol}, 0.1$ equiv.) in $\mathrm{CH}_{2} \mathrm{Cl}_{2}$ (4 mL) was stirred for $24 \mathrm{~h}$. Chromatography (35\% ethyl acetate-petroleum ether) yielded methyl (toluene-4-sulfonyl)pent-4-enoate $\mathbf{5 b}(80 \mathrm{mg}, 62 \%)$ as a colorless oil.

Method B: According to the general procedure, a mixture of tosylmalonate $\mathbf{4 b}$ (130 mg, 0.42 mmol), TBDMSOTf (200 $\mu \mathrm{L}, 0.87 \mathrm{mmol}, 2.1$ equiv.) and DBU (131 $\mu \mathrm{L}, 0.87 \mathrm{mmol}, 2.1$ equiv.) in $\mathrm{CH}_{2} \mathrm{Cl}_{2}(1 \mathrm{~mL})$ was stirred for $15 \mathrm{~min}$. Chromatography (25\% ethyl acetatepetroleum ether) yielded $\mathbf{5 b}(82 \mathrm{mg}, 73 \%)$ as a colorless oil.

$\mathrm{R}_{\mathrm{f}} 0.61$ (25\% ethyl acetate-petroleum ether); ${ }^{1} \mathrm{H} \mathrm{NMR} \delta 7.73(2 \mathrm{H}, \mathrm{d}, J 8.0 \mathrm{~Hz}, 2 \mathrm{H}$-ortho $\mathrm{Ts})$, $7.35(2 \mathrm{H}, \mathrm{d}, J 8.0 \mathrm{~Hz}, 2 \mathrm{H}-m e t a \mathrm{Ts}), 5.63\left(1 \mathrm{H}, \mathrm{m}, \mathrm{CH}=\mathrm{CH}_{2}\right), 5.09(1 \mathrm{H}, \mathrm{d}, J 18.0 \mathrm{~Hz}$, $\mathrm{CH}=\mathrm{CHaHb}), 5.07(1 \mathrm{H}, \mathrm{d}, J 9.5 \mathrm{~Hz}, \mathrm{CH}=\mathrm{CHaHb}), 3.98(1 \mathrm{H}, \mathrm{dd}, J 4.0,11.0 \mathrm{~Hz}, \mathrm{CH}-\mathrm{Ts}), 3.65$ $\left(3 \mathrm{H}, \mathrm{s}, \mathrm{OCH}_{3}\right), 2.70\left(2 \mathrm{H}, \mathrm{m}, \mathrm{CH}_{2}-\mathrm{CH}=\mathrm{CH}_{2}\right), 2.45\left(3 \mathrm{H}, \mathrm{s}, \mathrm{CH}_{3} \mathrm{Ts}\right)$.

\section{Methyl 3-ethyl-(toluene-4-sulfonyl)pent-4-enoate (5c)}

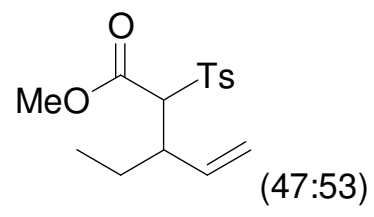

From $(E)$-methyl (2-pentenyl) (toluene-4-sulfonyl)malonate 4c

Method A: According to the general procedure, a mixture of tosylmalonate $4 \mathbf{c}$ (56 $\mathrm{mg}, 0.16$ mmol), BSA (24 $\mu \mathrm{L}, 0.16 \mathrm{mmol}, 1$ equiv.) and KOAc (2 mg, $0.02 \mathrm{mmol}, 0.1$ equiv.) in

\footnotetext{
${ }^{4}$ Ono, N.; Yoshimira, T.; Saito, T.; Tamura, R.; Tanikaga, R.; Kaji, A. Bull. Chem. Soc. Japan 1979, 52, 17161719.
} 
$\mathrm{CH}_{2} \mathrm{Cl}_{2}(2 \mathrm{~mL})$ was stirred for $24 \mathrm{~h}$. Chromatography (35\% ethyl acetate-petroleum ether) yielded methyl 3-ethyl-(toluene-4-sulfonyl)pent-4-enoate $\mathbf{5 c}(36 \mathrm{mg}, 74 \%)$ as a colorless oil.

Method B: According to the general procedure, a mixture of tosylmalonate 4c (103 mg, 0.31 mmol), TBDMSOTf (146 $\mu \mathrm{L}, 0.64$ mmol, 2.1 equiv.) and DBU (95 $\mu \mathrm{L}, 0.64 \mathrm{mmol}, 2.1$ equiv.) in $\mathrm{CH}_{2} \mathrm{Cl}_{2}(1 \mathrm{~mL})$ was stirred for $30 \mathrm{~min}$. Chromatography (35\% ethyl acetatepetroleum ether) yielded $\mathbf{5 c}(55 \mathrm{mg}, 61 \%)$ as a colorless oil.

\section{From (Z)-methyl (2-pentenyl) (toluene-4-sulfonyl)malonate 4d}

Method B: According to the general procedure, a mixture of tosylmalonate 4d (98 mg, 0.29 mmol), TBDMSOTf (139 $\mu \mathrm{L}, 0.61 \mathrm{mmol}, 2.1$ equiv.) and DBU (90 $\mu \mathrm{L}, 0.61 \mathrm{mmol}, 2.1$ equiv.) in $\mathrm{CH}_{2} \mathrm{Cl}_{2}(1 \mathrm{~mL})$ was stirred for $15 \mathrm{~min}$. Chromatography (25\% ethyl acetatepetroleum ether) yielded $\mathbf{5 c}(15 \mathrm{mg}, 18 \%)$ as a colorless oil.

$\mathrm{R}_{\mathrm{f}} 0.74$ (25\% ethyl acetate-petroleum ether); IR (film) $\mathrm{v}_{\mathrm{CO}} 1742 \mathrm{~cm}^{-1} ;{ }^{1} \mathrm{H}$ NMR $\delta 7.76$ (d, $J$ $8.0 \mathrm{~Hz}, 2 \mathrm{H}$-ortho Ts major isomer), 7.75 (d, J $8.0 \mathrm{~Hz}, 2 \mathrm{H}$-ortho Ts minor isomer), 7.33 (d, J $8.0 \mathrm{~Hz}, 2 \mathrm{H}$-meta Ts major isomer), 7.31 (d, J 8.0 Hz, 2H-meta Ts minor isomer), $5.55(1 \mathrm{H}, \mathrm{m}$, $\left.\mathrm{C} H=\mathrm{CH}_{2}\right), 5.10\left(2 \mathrm{H}, \mathrm{m}, \mathrm{CH}=\mathrm{CH}_{2}\right), 3.99$ (d, $J 10.0 \mathrm{~Hz}, \mathrm{CH}$-Ts minor isomer), 3.98 (d, $J 9.0$ $\mathrm{Hz}, \mathrm{CH}$-Ts major isomer), 3.63 (s, $\mathrm{OCH}_{3}$ minor isomer), 3.49 (s, $\mathrm{OCH}_{3}$ major isomer), 2.77 (qd, $J$ 3.0, $9.0 \mathrm{~Hz}, \mathrm{CH}-\mathrm{CH}=\mathrm{CH}_{2}$ major isomer), 2.67 (qd, 3.0, $10.0 \mathrm{~Hz}, \mathrm{CH}-\mathrm{CH}=\mathrm{CH}_{2}$ minor isomer), $2.43\left(3 \mathrm{H}, \mathrm{s}, \mathrm{CH}_{3} \mathrm{Ts}\right), 1.45$ (m, $\mathrm{CH}_{2}-\mathrm{CH}_{3}$ major isomer), 1.25 (m, $\mathrm{CH}_{2}-\mathrm{CH}_{3}$ minor isomer), 0.87 (t, $J 7.5 \mathrm{~Hz}, \mathrm{CH}_{2}-\mathrm{CH}_{3}$ major isomer), 0.81 (t, $J 7.5 \mathrm{~Hz}, \mathrm{CH}_{2}-\mathrm{CH}_{3}$ minor isomer); ${ }^{13} \mathrm{C}$ NMR $\delta 166.4$ and 166.1, 145.3, 136.4 and 136.1, 129.6, 129.4, 129.3, 128.9, 128.4, 118.8 and 118.6, 75.2 and 74.6, 52.7 and 52.5, 44.7 and 44.6, 25.2 and 25.1, 21.7, 11.2 and 11.0; MS (CI) m/z: $314\left[\mathrm{M}+\mathrm{NH}_{4}\right]^{+}$; HRMS (CI) $\mathrm{m} / \mathrm{z}$ calcd for $\left[\mathrm{C}_{15} \mathrm{H}_{20} \mathrm{O}_{4} \mathrm{~S}+\mathrm{NH}_{4}\right]^{+}: 314.1426$, found: 314.1415; Anal. calcd for $\mathrm{C}_{15} \mathrm{H}_{20} \mathrm{O}_{4} \mathrm{~S}$ : C, 60.79; H, 6.80; found: C, 60.87; H, 7.00.

\section{Methyl 3,3-dimethyl-(toluene-4-sulfonyl)pent-4-enoate (5e)}

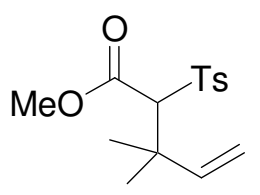

Method A: According to the general procedure, a mixture of tosylmalonate 4e (114 mg, 0.34 mmol), BSA ( $81 \mu \mathrm{L}, 0.34 \mathrm{mmol}, 1$ equiv.) and KOAc ( $3 \mathrm{mg}, 0.03 \mathrm{mmol}, 0.1$ equiv.) in $\mathrm{CH}_{2} \mathrm{Cl}_{2}(2 \mathrm{~mL})$ was stirred for $18 \mathrm{~h}$. Chromatography (25\% ethyl acetate-petroleum ether) 
yielded methyl 3,3-dimethyl-(toluene-4-sulfonyl)pent-4-enoate $\mathbf{5 e}(83 \mathrm{mg}, 80 \%)$ as a colorless oil.

Method B: According to the general procedure, a mixture of tosylmalonate $4 \mathbf{e}(121 \mathrm{mg}, 0.36$ mmol), TBDMSOTf (172 $\mu \mathrm{L}, 0.55 \mathrm{mmol}, 2.1$ equiv.) and DBU (112 $\mu \mathrm{L}, 0.75 \mathrm{mmol}, 2.1$ equiv.) in $\mathrm{CH}_{2} \mathrm{Cl}_{2}(1 \mathrm{~mL})$ was stirred for $15 \mathrm{~min}$. Chromatography (25\% ethyl acetatepetroleum ether) yielded $\mathbf{5 e}(75 \mathrm{mg}, 71 \%)$ as a colorless oil.

$\mathrm{R}_{\mathrm{f}} 0.63$ (25\% ethyl acetate-petroleum ether); IR (film) $v_{\mathrm{CO}} 1743 \mathrm{~cm}^{-1 ; 1} \mathrm{H}$ NMR $\delta 7.72(2 \mathrm{H}, \mathrm{d}$, $J 8.0 \mathrm{~Hz}, 2 \mathrm{H}$-ortho Ts), 7.30 (2H, d, J $8.0 \mathrm{~Hz}, 2 \mathrm{H}$-meta Ts), $6.01(1 \mathrm{H}, \mathrm{dd}, J 10.5,17.5 \mathrm{~Hz}$, $\left.\mathrm{C} H=\mathrm{CH}_{2}\right), 5.01(1 \mathrm{H}, \mathrm{d}, J 17.5 \mathrm{~Hz}, \mathrm{CH}=\mathrm{CHaHb}), 4.99(1 \mathrm{H}, \mathrm{dd}, J 1.0,17.5 \mathrm{~Hz}, \mathrm{CH}=\mathrm{CHa} H b)$, 3.96 (1H, s, CH-Ts), $3.46\left(3 \mathrm{H}, \mathrm{s}, \mathrm{OCH}_{3}\right), 2.41$ (3H, s, $\left.\mathrm{CH}_{3} \mathrm{Ts}\right), 1.37\left(3 \mathrm{H}, \mathrm{s}, \mathrm{CH}_{3}\right), 1.35$ (3H, s, $\left.\mathrm{CH}_{3}\right) ;{ }^{13} \mathrm{C}$ NMR $\delta$ 166.0, 145.1, 143.6, 136.9, 129.6, 129.1, 113.1, 78.7, 52.5, 41.0, 26.1, 25.1, 21.8; MS (CI) m/z: 426, 315, $314\left[\mathrm{M}+\mathrm{NH}_{4}\right]^{+}, 297[\mathrm{M}+\mathrm{H}]^{+}, 174 ;$ HRMS (CI) m/z calcd for $\left[\mathrm{C}_{15} \mathrm{H}_{20} \mathrm{O}_{4} \mathrm{~S}+\mathrm{NH}_{4}\right]^{+}$: 297.1161, found: 297.1158; Anal. calcd for $\mathrm{C}_{15} \mathrm{H}_{20} \mathrm{O}_{4} \mathrm{~S}: \mathrm{C}, 60.79 ; \mathrm{H}$, 6.80; found: C, 61.00; H, 6.96.

\section{Methyl 4-methyl-3-phenyl-(toluene-4-sulfonyl)pent-4-enoate (5f)}

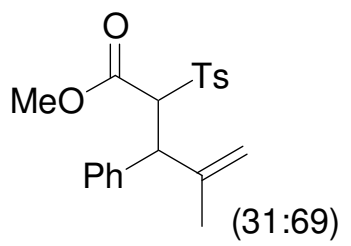

Method A: According to the general procedure, a mixture of tosylmalonate $4 \mathbf{f}(61 \mathrm{mg}, 0.15$ mmol), BSA (37 $\mu \mathrm{L}, 0.15 \mathrm{mmol}, 1$ equiv.) and KOAc (1.5 mg, $0.015 \mathrm{mmol}, 0.1$ equiv.) in $\mathrm{CH}_{2} \mathrm{Cl}_{2}(2 \mathrm{~mL})$ was stirred for $24 \mathrm{~h}$. Chromatography (35\% ethyl acetate-petroleum ether) yielded methyl 4-methyl-3-phenyl-(toluene-4-sulfonyl)pent-4-enoate $\mathbf{5 f}(30 \mathrm{mg}, 55 \%)$ as a colorless solid.

Method B: According to the general procedure, a mixture of tosylmalonate $4 \mathbf{f}$ (106 $\mathrm{mg}, 0.26$ mmol), TBDMSOTf (146 $\mu \mathrm{L}, 0.55 \mathrm{mmol}, 2.1$ equiv.) and DBU (83 $\mu \mathrm{L}, 0.55 \mathrm{mmol}, 2.1$ equiv.) in $\mathrm{CH}_{2} \mathrm{Cl}_{2}(1 \mathrm{~mL})$ was stirred for $15 \mathrm{~min}$. Chromatography (25\% ethyl acetatepetroleum ether) yielded $\mathbf{5 f}(55 \mathrm{mg}, 59 \%)$ as a colorless solid.

$\mathrm{R}_{\mathrm{f}} 0.59$ (25\% ethyl acetate-petroleum ether); IR (film) $v_{\mathrm{CO}} 1743 \mathrm{~cm}^{-1} ;{ }^{1} \mathrm{H}$ NMR $\delta 7.80(2 \mathrm{H}$, d, J $8.5 \mathrm{~Hz}, 2 \mathrm{H}$-ortho Ts), 7.32-7.00 (7H, m, 2H-meta Ts, 5H Ph), 5.09 (s, C=CHaHb minor isomer), 4.90 (s, $\mathrm{C}=\mathrm{CHaHb}$ major isomer ), 4.87 (s, $\mathrm{C}=\mathrm{CHaHb}$ minor isomer), 4.77 (d, $J 12.0$ 
$\mathrm{Hz}, \mathrm{CH}$-Ts minor isomer), 4.75 (s, $\mathrm{C}=\mathrm{CHaHb}$ major isomer), 4.74 (d, $J 12.0 \mathrm{~Hz}, \mathrm{CH}$-Ts major isomer), 4.10 (d, $J 12.0 \mathrm{~Hz}, \mathrm{CH}-\mathrm{Ph}$ minor isomer), 4.08 (d, $J 12.0 \mathrm{~Hz}, \mathrm{CH}-\mathrm{Ph}$ major isomer), 3.71 (s, $\mathrm{OCH}_{3}$ major isomer), 3.29 (s, $\mathrm{OCH}_{3}$ minor isomer), 2.44 (s, $\mathrm{CH}_{3}$ Ts minor isomer), 2.34 (s, $\mathrm{CH}_{3}$ Ts major isomer), 1.60 (s, $\mathrm{CH}_{3}$ major isomer), $1.57\left(\mathrm{~s}, \mathrm{CH}_{3}\right.$ minor isomer); ${ }^{13} \mathrm{C}$ NMR $\delta 166.0$ and 165.7, 145.8 and 145.4, 144.5, 142.6, 138.8, 136.9, 136.2, 135.0, 129.7, $129.5,129.4,129.1,128.5,128.3,127.9,127.4,114.8,111.4,74.5$ and 12.8, 53.2 and 52.7, 52.2 and 51.6, 21.9 and 19.5, 21.8 and 21.7; MS (CI) m/z: 734, 735, 377, $376\left[\mathrm{M}+\mathrm{NH}_{4}\right]^{+}, 359$ $[\mathrm{M}+\mathrm{H}]^{+}, 203$; HRMS (CI) $\mathrm{m} / \mathrm{z}$ calcd for $\left[\mathrm{C}_{20} \mathrm{H}_{22} \mathrm{O}_{4} \mathrm{~S}+\mathrm{NH}_{4}\right]^{+}:$376.1583, found: 376.1596; Anal. calcd for $\mathrm{C}_{20} \mathrm{H}_{22} \mathrm{O}_{4} \mathrm{~S}$ : C, 67.01; H, 6.19; found: C, 66.89; H, 6.22.

(1'R, 5'S)-Methyl (5-isopropenyl-2-methylenecyclohexyl)(toluene-4-sulfonyl)acetate and (1'S, 5'S)-methyl (5-isopropenyl-2-methylenecyclohexyl)(toluene-4-sulfonyl)acetate (5g)

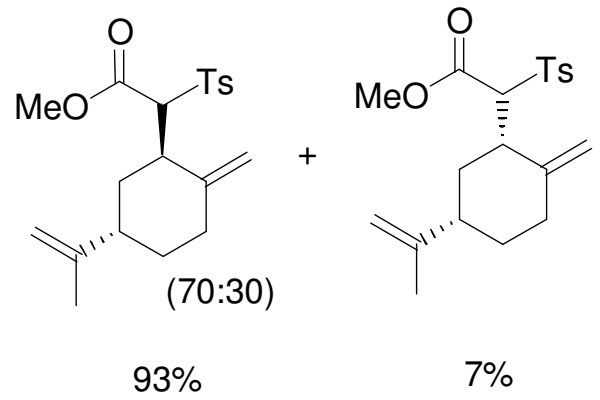

Method A: According to the general procedure, a mixture of tosylmalonate $4 \mathbf{g}$ (55 $\mathrm{mg}, 0.14$ mmol), BSA (33 $\mu \mathrm{L}, 0.14 \mathrm{mmol}, 1$ equiv.) and KOAc (1 mg, $0.014 \mathrm{mmol}, 0.1$ equiv.) in $\mathrm{CH}_{2} \mathrm{Cl}_{2}$ (4 mL) was stirred for $20 \mathrm{~h}$. Chromatography (35\% ethyl acetate-petroleum ether) yielded an inseparable mixture of (1'R, 5'S)-methyl (5-isopropenyl-2methylenecyclohexyl)(toluene-4-sulfonyl)acetate and (1'S, 5'S)-methyl (5-isopropenyl-2methylenecyclohexyl)(toluene-4-sulfonyl)acetate $\mathbf{5 g}$ (22 mg, 45\%) as a colorless solid.

$\mathrm{R}_{\mathrm{f}} 0.50$ (25\% ethyl acetate-petroleum ether); IR (film) $v_{\mathrm{CO}} 1743 \mathrm{~cm}^{-1} ;{ }^{1} \mathrm{H}$ NMR $\delta$ 7.81-7.74 (2H, m, 2H-ortho Ts), 7.35-7.26 (2H, m, 2H-meta Ts), 4.95-4.60 (4H, m, $\left.2 \mathrm{C}=\mathrm{CH}_{2}\right), 4.51$ (d, $J 11.5 \mathrm{~Hz}, \mathrm{CH}-\mathrm{Ts}$ minor (1'R, 5' $S$ ) compound), 4.47 (d, $J 12.0 \mathrm{~Hz}, \mathrm{CH}-\mathrm{Ts}$ major $(1 R, 5 S)$ compound), 4.19 (d, J 11.5 Hz, CH-Ts (1'S, 5'S) compound), 3.44-2.90 (m, 1H), 2.72-2.45 (m, 1H), $2.43\left(3 \mathrm{H}, \mathrm{s}, \mathrm{CH}_{3} \mathrm{Ts}\right), 2.40-1.72(\mathrm{~m}, 4 \mathrm{H}), 1.71$ (s, $\mathrm{CH}_{3}$ major (1'R, 5' $S$ ) compound), 1.60 (s, $\mathrm{CH}_{3}$ minor (1'R, 5' $S$ ) compound), 1.59-1.10 (m, 2H); ${ }^{13} \mathrm{C}$ NMR $\delta 166.3$ and 166.2, 148.9 and $148.5,135.4,129.7,129.6,129.3,113.7,112.4,109.5$ and 109.2, 53.0 and 52.7 and 52.6, 42.9 and 42.3, 40.0 and 39.5, 38.7, 37.2, 37.1, 37.0, 33.9, 33.0, 32.6, 31.1, 30.6, 21.8 
and 21.1 and 20.5; MS (CI) m/z: $380\left[\mathrm{M}+\mathrm{NH}_{4}\right]^{+}$, 134; HRMS (CI) m/z calcd for $\left[\mathrm{C}_{20} \mathrm{H}_{26} \mathrm{O}_{4} \mathrm{~S}+\mathrm{NH}_{4}\right]^{+}:$380.1896, found: 380.1888; Anal. calcd for $\mathrm{C}_{20} \mathrm{H}_{26} \mathrm{O}_{4} \mathrm{~S}: \mathrm{C}, 66.27 ; \mathrm{H}$, 7.23; found: C, 66.39; H, 7.20.

(1'R, 3'S, 5'R)-Methyl (6,6-dimethyl-2-methylenebicyclo[3.1.1]hept-3-yl)(toluene-4sulfonyl)acetate $(5 \mathrm{~h})$

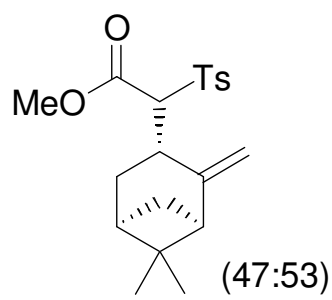

Method A: According to the general procedure, a mixture of tosylmalonate $4 \mathbf{h}(82 \mathrm{mg}, 0.20$ mmol), BSA (49 $\mu \mathrm{L}, 0.20 \mathrm{mmol}, 1$ equiv.) and KOAc (2 mg, $0.02 \mathrm{mmol}, 0.1$ equiv.) in $\mathrm{CH}_{2} \mathrm{Cl}_{2}(2 \mathrm{~mL})$ was stirred for $18 \mathrm{~h}$. Chromatography (25\% ethyl acetate-petroleum ether) yielded (1'R, 3'S, 5'R)-methyl (6,6-dimethyl-2-methylenebicyclo[3.1.1]hept-3-yl)(toluene-4sulfonyl)acetate $\mathbf{5 h}(53 \mathrm{mg}, 72 \%)$ as a colorless oil.

Method B: According to the general procedure, a mixture of tosylmalonate $\mathbf{4 h}$ (72 $\mathrm{mg}, 0.18$ mmol), TBDMSOTf ( $85 \mu \mathrm{L}, 0.37 \mathrm{mmol}, 2.1$ equiv.) and DBU (56 $\mu \mathrm{L}, 0.37 \mathrm{mmol}, 2.1$ equiv.) in $\mathrm{CH}_{2} \mathrm{Cl}_{2}(1 \mathrm{~mL})$ was stirred for $15 \mathrm{~min}$. Chromatography (25\% ethyl acetate-petroleum ether) yielded $\mathbf{5 h}(49 \mathrm{mg}, 76 \%)$ as a colorless oil.

$\mathrm{R}_{\mathrm{f}} 0.76$ (25\% ethyl acetate-petroleum ether); IR (film) $v_{\mathrm{CO}} 1742 \mathrm{~cm}^{-1} ;{ }^{1} \mathrm{H}$ NMR $\delta 7.81$ (d, $J$ $8.0 \mathrm{~Hz}, 2 \mathrm{H}$-ortho Ts one isomer), 7.80 (d, J 8.5 Hz, 2H-ortho Ts other isomer), 7.35 (d, J 8.5 $\mathrm{Hz}, 2 \mathrm{H}-m e t a \mathrm{Ts}$ one isomer), 7.34 (d, J $8.0 \mathrm{~Hz}, 2 \mathrm{H}-m e t a$ Ts other isomer), 4.79 (dd, J 2.0, $10.0 \mathrm{~Hz}, \mathrm{C}=\mathrm{CH}_{2}$ major isomer), 4.63 (s, $\mathrm{C}=\mathrm{CH}_{2}$ minor isomer), 4.35 (d, J 3.5 Hz, $\mathrm{CH}$-Ts major isomer), 4.25 (d, J $11.0 \mathrm{~Hz}, \mathrm{CH}$-Ts minor isomer), 3.60 (m, $\mathrm{CH}$-CH-Ts), 3.58 (s, $\mathrm{OCH}_{3}$ major isomer), 3.46 (s, $\mathrm{OCH}_{3}$ minor isomer), 3.21 (m, $\left.\mathrm{CH}-\mathrm{CH}-\mathrm{Ts}\right), 2.60-1.90(5 \mathrm{H}, \mathrm{m}), 2.44$ (3H, s, $\mathrm{CH}_{3} \mathrm{Ts}$ ), 1.41 (d, J 10.5 Hz, $\mathrm{CH}$ one isomer), 1.22 (s, $\mathrm{CH}_{3}$ one isomer), 1.21 (s, $\mathrm{CH}_{3}$ 
other isomer), 0.82 (d, $J 10.0 \mathrm{~Hz}, \mathrm{CH}$ other isomer), 0.78 (s, $\mathrm{CH}_{3}$ one isomer), $0.78\left(\mathrm{~s}, \mathrm{CH}_{3}\right.$ one isomer), 0.64 (s, $\mathrm{CH}_{3}$ other isomer); ${ }^{13} \mathrm{C} \mathrm{NMR} \delta 166.7$ and 166.0, 151.7, 149.3, 145.3, 135.5 and 134.8, 129.8, 129.7, 129.5, 129.3, 112.7 and 109.9, 80.1 and 75.6, 52.6, 52.3, 52.2, 52.0, 41.0, 40.7, 40.6, 40.0, 34.1 and 32.7, 28.9, 27.6, 26.9, 26.1, 26.0, 25.7, 21.9, 21.8, 21.7; MS (CI) m/z: $380 \quad\left[\mathrm{M}+\mathrm{NH}_{4}\right]^{+}, \quad 363 \quad[\mathrm{M}+\mathrm{H}]^{+}, \quad 209 ; \quad H R M S \quad(\mathrm{CI}) \mathrm{m} / \mathrm{z}$ calcd for $\left[\mathrm{C}_{20} \mathrm{H}_{26} \mathrm{O}_{4} \mathrm{~S}+\mathrm{NH}_{4}\right]^{+}:$380.1896, found: 380.1907; Anal. calcd for $\mathrm{C}_{20} \mathrm{H}_{26} \mathrm{O}_{4} \mathrm{~S}: \mathrm{C}, 66.27 ; \mathrm{H}$, 7.23; found: C, 66.08; $\mathrm{H}, 7.15$.

\section{(E)-Methyl 3-methyl-(toluene-4-sulfonyl)hex-4-enoate (5i)}

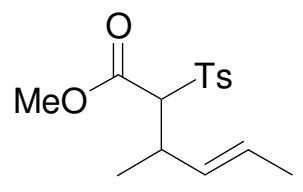

$(47: 53)$

Method A: According to the general procedure, a mixture of tosylmalonate $4 \mathbf{i}$ (86 $\mathrm{mg}, 0.25$ mmol), BSA (61 $\mu \mathrm{L}, 0.25 \mathrm{mmol}, 1$ equiv.) and KOAc (3 mg, $0.025 \mathrm{mmol}, 0.1$ equiv.) in $\mathrm{CH}_{2} \mathrm{Cl}_{2}(2 \mathrm{~mL})$ was stirred for $15 \mathrm{~h}$. Chromatography (25\% ethyl acetate-petroleum ether) yielded (E)-methyl 3-methyl-(toluene-4-sulfonyl)hex-4-enoate $\mathbf{5 i}(66 \mathrm{mg}, 88 \%)$ as a colorless oil.

Method B: According to the general procedure, a mixture of tosylmalonate 4i (139 mg, 0.41 mmol), TBDMSOTf (197 $\mu \mathrm{L}, 0.86 \mathrm{mmol}, 2.1$ equiv.) and DBU (128 $\mu \mathrm{L}, 0.86 \mathrm{mmol}, 2.1$ equiv.) in $\mathrm{CH}_{2} \mathrm{Cl}_{2}(1.5 \mathrm{~mL})$ was stirred for $15 \mathrm{~min}$. Chromatography (25\% ethyl acetatepetroleum ether) yielded $\mathbf{5 i}(100 \mathrm{mg}, 83 \%)$ as a colorless oil.

$\mathrm{R}_{\mathrm{f}} 0.67$ (25\% ethyl acetate-petroleum ether); IR (film) $v_{\mathrm{CO}} 1743 \mathrm{~cm}^{-1} ;{ }^{1} \mathrm{H}$ NMR $\delta 7.74$ (d, $J$ $8.5 \mathrm{~Hz}, 2 \mathrm{H}$-ortho Ts one isomer), 7.71 (d, $J 8.5 \mathrm{~Hz}, 2 \mathrm{H}$-ortho Ts other isomer), 7.31 (d, J 8.0 $\mathrm{Hz}, 2 \mathrm{H}$-meta Ts one isomer), 7.30 (d, J 8.0 Hz, 2H-meta Ts other isomer), 5.49 (qd, J 2.0, 6.0 $\mathrm{Hz}, \mathrm{CH}=\mathrm{CH}-\mathrm{Me}$ one isomer), 5.43 (qd, $J$ 2.0, $6.5 \mathrm{~Hz}, \mathrm{CH}=\mathrm{CH}-\mathrm{Me}$ other isomer), 5.24 (m, $\mathrm{CH}=\mathrm{CH}$-Me one isomer), 5.19 (m, $\mathrm{CH}=\mathrm{CH}$-Me other isomer), 3.86 (d, J 14.0 Hz, CH-Ts one isomer), 3.83 (d, $J 15.5 \mathrm{~Hz}, \mathrm{CH}$-Ts other isomer), 3.64 (s, $\mathrm{OCH}_{3}$ one isomer), 3.47 (s, $\mathrm{OCH}_{3}$ other isomer), $2.93\left(1 \mathrm{H}, \mathrm{m}, \mathrm{CH}-\mathrm{CH}_{3}\right), 2.42\left(3 \mathrm{H}, \mathrm{s}, \mathrm{CH}_{3} \mathrm{Ts}\right), 1.59$ (dd, J 1.0, 6.0 Hz, CH-CH one isomer), 1.55 (dd, $J$ 1.5, $6.5 \mathrm{~Hz}, \mathrm{CH}-\mathrm{CH}_{3}$ other isomer), 1.28 (d, $J 6.5 \mathrm{~Hz}, \mathrm{CH}=\mathrm{CH}-\mathrm{CH}_{3}$ one isomer), 1.02 (d, $J 6.5 \mathrm{~Hz}, \mathrm{CH}=\mathrm{CH}-\mathrm{CH}_{3}$ other isomer); ${ }^{13} \mathrm{C} \mathrm{NMR} \delta 166.3,145.3$ and 
145.2, 135.6 and 135.4, 131.5 and 130.8, 129.7, 129.5, 129.3, 127.9 and 127.3, 76.3 and 75.8,

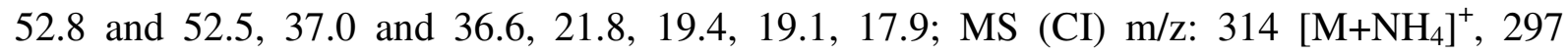
$[\mathrm{M}+\mathrm{H}]^{+}, 141$; HRMS (CI) $\mathrm{m} / \mathrm{z}$ calcd for $\left[\mathrm{C}_{15} \mathrm{H}_{20} \mathrm{O}_{4} \mathrm{~S}+\mathrm{NH}_{4}\right]^{+}:$297.1161, found: 297.1159; Anal. calcd for $\mathrm{C}_{15} \mathrm{H}_{20} \mathrm{O}_{4} \mathrm{~S}: \mathrm{C}, 60.79 ; \mathrm{H}, 6.80$; found: C, 60.84; H, 6.91.

(1'S, 5'S)-Methyl (5-isopropenyl-2-methyl-cyclohex-2-enyl)(toluene-4-sulfonyl)acetate $(5 \mathbf{j})$

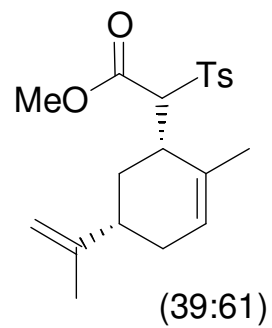

Method A: According to the general procedure, a mixture of tosylmalonate 4j (60 mg, 0.15 mmol), BSA (36 $\mu \mathrm{L}, 0.15 \mathrm{mmol}, 1$ equiv.) and KOAc (1.5 mg, $0.015 \mathrm{mmol}, 0.1$ equiv.) in $\mathrm{CH}_{2} \mathrm{Cl}_{2}(1.5 \mathrm{~mL})$ was stirred for $15 \mathrm{~h}$. Chromatography (25\% ethyl acetate-petroleum ether) yielded (1'S, 5'S)-methyl (5-isopropenyl-2-methyl-cyclohex-2-enyl)(toluene-4sulfonyl)acetate $\mathbf{5 j}$ (10 $\mathrm{mg}, 19 \%)$ as a colorless oil.

Method B: According to the general procedure, a mixture of tosylmalonate $\mathbf{4 j}$ (118 mg, 0.29 mmol), TBDMSOTf (140 $\mu \mathrm{L}, 0.61 \mathrm{mmol}, 2.1$ equiv.) and DBU (91 $\mu \mathrm{L}, 0.61 \mathrm{mmol}, 2.1$ equiv.) in $\mathrm{CH}_{2} \mathrm{Cl}_{2}(1.5 \mathrm{~mL})$ was stirred for $15 \mathrm{~min}$. Chromatography (25\% ethyl acetatepetroleum ether) yielded $\mathbf{5 j}$ (60 $\mathrm{mg}, 57 \%)$ as a colorless oil.

$\mathrm{R}_{\mathrm{f}} 0.61$ (25\% ethyl acetate-petroleum ether); IR (film) $v_{\mathrm{CO}} 1747 \mathrm{~cm}^{-1} ;{ }^{1} \mathrm{H}$ NMR $\delta 7.79(2 \mathrm{H}$, m, 2H-ortho Ts), 7.31 (2H, m, 2H-meta Ts), 5.58 (bs, $\mathrm{CH}=\mathrm{C}$ major isomer), 5.51 (bs, $\mathrm{CH}=\mathrm{C}$ minor isomer), $4.69\left(2 \mathrm{H}, \mathrm{m}, \mathrm{CH}_{2}=\mathrm{C}\right), 4.32$ (d, J $3.0 \mathrm{~Hz}, \mathrm{CH}$-Ts major isomer), 4.25 (d, J 4.5 $\mathrm{Hz}, \mathrm{CH}$-Ts minor isomer), 3.60 ( $\mathrm{s}, \mathrm{OCH}_{3}$ major isomer), 3.56 (s, $\mathrm{OCH}_{3}$ minor isomer), 3.49$3.41(1 \mathrm{H}, \mathrm{m}), 3.15(1 \mathrm{H}, \mathrm{bs}), 2.43\left(3 \mathrm{H}, \mathrm{s}, \mathrm{CH}_{3} \mathrm{Ts}\right), 2.40-1.40(10 \mathrm{H}, \mathrm{m}) ;{ }^{13} \mathrm{C} \mathrm{NMR} \delta 166.8$ and 165.3, 148.8, 145.3, 136.1, 131.8, 130.7, 129.8, 129.6, 129.1, 127.1, 126.3, 109.2, 71.8 and 70.6, 52.7 and 52.5, 42.0, 41.7, 41.2, 40.0, 31.7, 30.8, 30.5, 30.1, 25.8, 21.9, 21.8, 21.1, 20.8; 
MS (CI) m/z: $380\left[\mathrm{M}+\mathrm{NH}_{4}\right]^{+}, 226,207,174,152,135,52$; HRMS (CI) $\mathrm{m} / \mathrm{z}$ calcd for $\left[\mathrm{C}_{20} \mathrm{H}_{26} \mathrm{O}_{4} \mathrm{~S}+\mathrm{NH}_{4}\right]^{+}: 380.1896$, found: 380.1877 ; Anal. calcd for $\mathrm{C}_{20} \mathrm{H}_{26} \mathrm{O}_{4} \mathrm{~S}: \mathrm{C}, 66.27 ; \mathrm{H}$, 7.23; found: $\mathrm{C}, 66.41 ; \mathrm{H}, 7.09$. 